\title{
La postura de la Unión Europea frente al separatismo en Cataluña
}

\author{
A.V. Makárycheva
}

\section{$\rightarrow$ Resumen}

Las tendencias separatistas son más características de la Unión Europea (UE) que del resto del mundo. Este hecho se explica ante todo por la crisis de los valores europeos, los elementos religiosos y las nuevas prioridades. El propósito tanto de Cataluña como del País Vasco de dialogar directamente con Bruselas sin la participación de Madrid conlleva múltiples dificultades: el descontento y las advertencias del Gobierno de España representan un obstáculo dada la necesidad de la Unión Europea de tener en cuenta la postura de las autoridades oficiales de sus Estados miembros. A pesar de que las regiones han adquirido mayor protagonismo en la agenda de la Unión Europea, la política de esta comunidad sigue de cerca la situación de las autonomías y no es partidaria del separatismo. La UE intenta limitar la actividad y la conversión de las autonomías en estados independientes, utilizando para ello medidas institucionales. Además, el nuevo Estado independiente no entraria en el espacio europeo porque dejaria de formar parte de la UE y, por tanto, se vería obligado a resolver una serie de cuestiones, como la necesidad de crear una nueva moneda y lidiar con la posible crisis económica que se derive de ello, además del previsible descontento por la disminución de la calidad de vida y la conmoción social. Todos estos factores influyen en las autonomías a la hora de valorar las posibles consecuencias de sus actos, antes de tomar la decisión concreta sobre un referéndum. Cabe mencionar que tampoco es sencillo hacer un referéndum de estas caracteristicas porque no está contemplado en la Constitución de España.

\section{$\rightarrow$ Palabras clave}

Nacionalismo catalán, Unión Europea, procesos de integración, referéndum en Cataluña, separatismo en España, desintegración europea

Declaración de divulgación: El autor no informó de ningún posible conflicto de intereses. https://doi.org/10.46272/24093416-2020-8-2-65-75

\section{Artículo de investigación}

Ánna Vladimirovna Makárycheva, doctorada en politología, investigadora del Laboratorio de Análisis de Procesos Internacionales, Profesora del Departamento de Español, Instituto Estatal de Relaciones Internacionales de Moscú (MGIMO-Universidad) (Rusia)

\section{E-mail:}

makarycheva.a.v@my.mgimo.ru

Para la correspondencia: 119454, Rusia, Moscú, av.Vernadskogo, 76

Para citar: Makárycheva, Ánna Vladímirovna. "La postura de la Unión Europea frente al separatismo en Cataluña" [The position of the European Union towards separatism in Catalonia]. Cuadernos Iberoamericanos 8, no. 2 (2020): 65-75. https://doi. org/10.46272/2409-3416-20208-2-65-75. [In Spanish]

El artículo fue recibido por los editores: 02.09.2020

Aceptado para publicación: 05.10.2020 
El presente artículo aborda los problemas del nacionalismo catalán, sus premisas y la posterior reacción de la Unión Europea frente al referéndum de Cataluña y al movimiento independentista en esta autonomía. El objetivo principal del autor consiste no sólo en evaluar la situación actual del objeto de estudio, sino también en realizar un análisis histórico del nacionalismo en Cataluña para comprender la dinámica del desarrollo de este proceso. El tema del separatismo ha ganado actualidad en los últimos años a raíz del referéndum de Escocia del año 2014, que influyó notablemente en la decisión de las autoridades catalanas de organizar su propio referéndum, y los acontecimientos que hemos observado en Reino Unido, que el 31 de enero de 2020 abandonó formalmente la Unión Europea después de numerosas rondas de negociaciones.'

En el marco del estudio actual es importante examinar los puntos de vista de diversos autores dedicados a la relación del separatismo y la Unión Europea. Por ejemplo, J. Weiler considera que los movimientos separatistas son incompatibles con el proceso de integración europea. ${ }^{2}$ Desde este punto de vista es comprensible la postura de la Unión Europea respecto al separatismo, es decir, el rechazo de cualquier tipo de secesionismo y la afirmación de que el Estado que pudiera alcanzar la independencia no sería la parte de la Unión.

Por otra parte, no podemos olvidarnos del derecho internacional, donde se refleja la integridad de las fronteras y también el derecho a la autodeterminación. Ambos conceptos quedaron plasmados en el Acta Final de la Conferencia sobre Seguridad y Cooperación en Europa (Helsinki, 1975). ${ }^{3}$ En este contexto, N. Walker considera que los tratados de la Unión Europea "destilan una sensibilidad hacia el hecho regional" que dificulta la aplicación del concepto de autodeterminación. ${ }^{4}$ El autor subraya que la UE debe ser neutral, tratar a todos los movimientos secesionistas por igual y no influir el resultado final. ${ }^{5}$

La tercera postura es contraria y mantiene que la Unión Europea debe apoyar los movimientos separatistas puesto que son una expresión del principio democrático. En estas líneas, si algún Estado rechaza la organización y la realización de un referéndum por la independencia, la Unión debe apoyar a la provincia o comunidad que desee independizarse. ${ }^{6}$ En cualquier caso, la UE debe reaccionar de alguna manera a los acontecimientos y no hacer caso omiso de lo que ocurre en sus Estados miembros. En este contexto, los valores y principios relevantes europeos pueden desempeñar un papel muy importante. A continuación, analizamos la postura actual de la Unión Europea frente a Cataluña.

\section{El fenómeno del nacionalismo catalán: perspectiva histórica}

Cabe señalar que el origen del nacionalismo catalán se pierde en la noche de los tiempos, si consideramos que esta región del país gozó durante mucho tiempo de los privilegios y derechos civiles de una amplia autonomía. Cataluña obtuvo estos privilegios cuando pasó a formar parte del Reino de España tras el matrimonio dinástico de Isabel I de Castilla y Fernando de Aragón. Poco a poco, la independencia de la autonomía se vio disminuida y limitada, un proceso que culminó cuando, a raíz de La Guerra de Sucesión Española de 1714, se diluyó prácticamente

1 "Brexit: salida de Reino Unido de la UE," EUR-Lex, accessed October 1, 2020, https://eur-lex.europa.eu/content/news/Brexit-UK-withdrawal-from-the-eu.html?locale=es.

2 Weiler 2017, 12-31.

3 "Helsinki Final Act," Organization for Security and Co-operation in Europe, accessed October 1, 2020, https://www.osce.org/helsinki-final-act.

4 Walker 2017, 45-47.

5 lbid.

6 Nico Krisch, "The Spanish constitutional crisis: Law, legitimacy and popular sovereignty in question," Verfassungsblog: On Matters Constitutional, October 7, 2017, https://verfassungsblog. de/the-spanish-constitutional-crisis-law-legitimacy-and-popular-sovereignty-in-question/. 
la frágil independencia de la región. ${ }^{1}$ Por tanto, el siglo XVIII fue el siglo de la integración progresiva de Cataluña en la vida política y económica de España. ${ }^{2}$ Lamentablemente, este proceso integrador no contribuyó a la asimilación de los catalanes dada la complejidad del aparato burocrático. A diferencia de lo ocurrido en Francia, por ejemplo, las autoridades españolas no pudieron crear un estado unitario, sino que conservaron formalmente el estatus de federación. En la segunda mitad del siglo XIX, con un imperio español debilitado que ya había perdido su grandeza pasada, una nueva oleada de nacionalismos se extendió por el país.

El movimiento nacionalista actual surgió en Cataluña con un carácter claro y evidente a principios del siglo XX, cuando los partidarios de la independencia de Cataluña lograron que se aprobara el estatuto de la Mancomunidad de Cataluña y establecieron su propio partido: La Liga Regionalista. Este partido recibió amplio apoyo del público y luchó por conseguir mayor capacidad legislativa en la región, pese a que en aquel momento no se contemplaba la cuestión de la independencia. Durante la Guerra Civil Española, Cataluña, que de nuevo tenía como objetivo principal la independencia, apoyó al gobierno central republicano enfrentado a Francisco Franco. Finalizada la guerra, Franco tomó represalias y eliminó los estatutos de las autonomías, además de prohibir el idioma catalán y la cultura catalana. El régimen de la dictadura contribuyó en gran medida al crecimiento del nacionalismo en la región debido a la política de aquel tiempo, muy negativa para las tradiciones étnicas, culturales e históricas de Cataluña. En 1978, con la aprobación y ratificación de la Constitución española, la comunidad catalana recupera el estatus de república federativa y la lengua catalana obtiene reconocimiento oficial.

Es importante subrayar que, previamente, el movimiento nacionalista se limitaba a reclamar una ampliación de derechos de la región, siempre como parte integrante del país. Sin embargo, el objetivo actual es la independencia de Cataluña. ${ }^{3}$ Históricamente, este tipo de tendencias se afianzan cuando gozan de una situación geográfica favorable y otras características beneficiosas. Durante muchos siglos, Cataluña desempeñó un papel muy importante en el comercio exterior y también en la conformación y el fortalecimiento del propio Reino de España.

Las tendencias separatistas actuales se explican en gran medida por el hecho de que tanto Cataluña como el País Vasco son los centros industriales más importantes del país y las comunidades más desarrolladas en términos generales. En otras regiones, como Andalucia, Extremadura, Murcia o Castilla-La Mancha, las condiciones para el desarrollo de la agricultura son muy desfavorables debido a la sequedad del clima. Por tanto, las provincias españolas menos desarrolladas necesitan contribuciones que, básicamente, reciben del País Vasco y Cataluña. El descontento de estas comunidades con esta situación, es decir, con el uso de sus recursos para garantizar el bienestar económico de otras regiones del país, nutre las tendencias separatistas. Según los datos del Centro de Documentación y Estudios de San Sebastián, ${ }^{4}$ el País Vasco destina el 3,1\% de su PIB (producto interior bruto) a ayudas sociales, frente al 0,3\% que se destina en España. Los vascos, con una población de 2,1 millones de personas, aportan alrededor de 450 millones de euros al presupuesto nacional, mientras que los catalanes, con una población de 7,5 millones de personas, aportan menos

1 Добров Д. Состоится ли независимость Каталонии? [Электронный ресурс] // ИноСмИ. 1 августа 2014 г.

URL: https://inosmi.ru/world/20140801/222084465.html (дата обращения: 01.10.2020).

2 Solé Tura 1970, 23-25.

3 Хенкин С.М. Испания: испытание Каталонией [Электронный ресурс] // Сетевое издание Центра исследований и аналитики Фонда исторической перспективы. 14 января 2013 г. URL: http://www.perspektivy.info/oykumena/europe/ispanija_ispytanije_ katalonijej_2013-01-14.htm (дата обращения: 01.10.2020).

4 Centro de Documentación y Estudios de San Sebastián, accessed October 1, 2020, http://www.siis.net/. 
de 173 millones de euros, según datos del investigador Joan Subirats, director del Instituto de Gobierno y Políticas Públicas de la Universidad Autónoma de Barcelona. ${ }^{1}$ Basándonos en estos datos estadísticos, podemos concluir que la brecha en el desarrollo de las regiones resulta evidente y que las dos comunidades más desarrolladas del país no están dispuestas a sostener económicamente a toda España.

\section{Siglo XXI: continúan los problemas}

Exponemos brevemente los acontecimientos más relevantes del siglo XXI a este respecto y sus posibles consecuencias. La crisis financiera del año 2008 agravó las contradicciones existentes: el descontento de los catalanes por la obligación de financiar a toda España y la inestabilidad económica general reavivaron el movimiento separatista en Cataluña, expresado en términos pacíicos, a diferencia del País Vasco, donde la organización terrorista nacionalista ETA renunció a la lucha armada tan sólo en el año 2011.

Cabe mencionar el conocido proyecto del Estatuto de Autonomía de Cataluña de 2006, en cuyo preámbulo se define a Cataluña como nación, una declaración contraria a lo estipulado en la Constitución española. El proyecto del Estatuto originó el descontento de Madrid. La oposición más intensa la llevó a cabo el Partido Popular, impugnando 187 artículos. ${ }^{2}$ A raíz de las elecciones autonómicas de 2012, el Parlamento de Cataluña se conformó con una mayoría de diputados que apoyaban el independentismo catalán. En 2014, el deseo de celebrar el referéndum sobre la independencia tropezó con una fuerte resistencia jurídica por parte del Tribunal Constitucional de España y del Congreso de los Diputados, que se referían a la imposibilidad de celebrar un referéndum de estas características en el marco de la Constitución actual. Por esta razón, la Generalidad de Cataluña decidió anular el referéndum y sustituirlo por una consulta participativa que fue celebrada el 9 de noviembre de 2014. Esta consulta no tenía ningún peso juridico y estaba prohibida por las autoridades oficiales de España.

En 2015 se celebraron en Cataluña nuevas elecciones anticipadas, en las que los partidos separatistas obtuvieron la mayoría de los escaños, aunque el porcentaje de los votos obtenidos por estos partidos no alcanzó el 50 por ciento de los sufragios. ${ }^{3}$ El 5 de noviembre de 2015, el Parlamento de Cataluña aprobó una resolución en la que se proclamaba el inicio del proceso separatista de España. Los líderes de los partidos ganadores declararon la intención de crear una hoja de ruta para la independencia de la región que incluía una nueva constitución y nuevas estructuras estatales. En diciembre de ese mismo año, el Tribunal Constitucional de España declaró inconstitucional la resolución sobre la independencia en Cataluña. ${ }^{4}$

Finalmente, el 1 de octubre de 2017 se celebró el referéndum sobre la independencia sin el reconocimiento de las autoridades españolas. Según los datos de la Generalidad, el $90,2 \%$ de los votantes se pronunció a favor de la independencia y el 7,8\% en contra. ${ }^{5}$ Sin embargo, estos resultados no reflejan el equilibrio real de fuerzas dado que sólo participó el $43 \%$ del electorado con derecho a voto. La situación actual concluye con una victoria rotunda de las autoridades españolas. ${ }^{6}$ Además, hay que subrayar que Cataluña debió

1 "Joan Subirats. Universidad de Barcelona," Coursera, accessed October 1, 2020, https://www.coursera.org/instructor/joan-subirats-h.

2 Хенкин 2013.

3 Khenkin 2018.

4 Суд Испании признал отделение Каталонии неконституционным [Электронный ресурс] // ВBC News. Русская служба. 2 декабря 2015 г. URL: https://www.bbc.com/russian/news/2015/12/151202 spain_catalonia_ruling (дата обращения: 01.10.2020).

5 "El 90.18\% votó sí y la participación fue del 43\%, según datos definitivos," EITB, October 7, 2017, https://www.eitb.eus/es/noticias/politica/detalle/5129009/resultados-definitivos-referendumcataluna-1-octubre-2017/. 
afrontar una serie de problemas serios como consecuencia del referéndum realizado, de los cuales el más importante fue la aplicación, por parte del gobierno de España, del artículo 155 de la Constitución, según el cual la gestión de la comunidad autónoma pasa a manos del gobierno central de Madrid. ${ }^{1}$ Sin embargo, la llegada del nuevo gobierno autonómico encabezado por Quim Torra en junio de 2018 puso fin a esta situación. ${ }^{2}$

En cuanto al electorado, la brecha creciente no sólo existe entre el Gobierno de Cataluña y las autoridades españolas, sino también dentro de la propia sociedad catalana. Según los datos de los sociólogos, los sectores más pudientes de la sociedad, así como la población rural, los emigrantes de otras partes de España y los ciudadanos cuya lengua materna es el catalán son los más partidarios de la separación. ${ }^{3}$

\section{La postura de la Unión Europea}

Analizamos a continuación la postura de la Unión Europea frente al nacionalismo en general y a los acontecimientos de Cataluña en particular. Los documentos de la Unión Europea expresan con frecuencia que "las regiones son órganos operativos y tejido institucional de la Unión."4 En 1988, el Parlamento Europeo adoptó La Carta Europea de Autonomía Local. La retórica de la modernización de los estados, incluso de su regionalización, se inscribe en las nociones de la Unión Europea sobre los estados modernos, su funcionamiento y su desarrollo. Los elementos de federalización de Europa quedaron garantizados en el Tratado de la UE (Tratado de Maastricht) y en el Tratado de Lisboa. Hoy en día, la Unión Europea es una unión de estados que han delegado parte de sus poderes a un nivel supranacional, razón por la cual están obligados a tomar en cuenta la postura de la UE. ${ }^{5}$ El Tratado de Maastricht no sólo permitió crear un mercado común de bienes y servicios, una moneda única y una condición de ciudadanía, sino que también contribuyó a dar mayor protagonismo a las regiones de la Unión Europea, que desde entonces se consideran actores de la Unión. De este modo, las regiones pueden presentar enmiendas y recomendaciones en el Consejo de Europa y en la Comisión Europea sobre asuntos directamente relacionados con sus intereses. A nuestro modo de ver, la eficiencia de esta cooperación es discutible puesto que la Unión Europea considera, en primer lugar, la postura de los Estados miembros ante lo que denomina "el descontento de Cataluña y el País Vasco", las regiones más avanzadas de España. Estas dos regiones, junto con Navarra y Galicia, abogaron por la posibilidad de defender sus propios intereses directamente en Bruselas. Para ello se crearon organizaciones especiales, como el Comité Europeo de las Regiones, aunque tampoco esto significa que haya carta blanca puesto que su eficacia queda comprometida debido al carácter recomendatorio y asesor de la organización. La postura del gobierno central de Madrid hacia este tipo de iniciativas es clara: a las estructuras administrativas de España les preocupa la posibilidad de ampliar los poderes de sus regiones y temen una nueva oleada de separatismos, no sólo en el país sino en toda Europa, si se permitiera un diálogo directo entre las autonomías y Bruselas.

A pesar de la federalización formalmente establecida, la Unión Europea no apoya el fenómeno del separatismo y observa con atención los acontecimientos de Cataluña, por no mencionar el inédito referéndum de Reino Unido y los acontecimientos de enero de 2020. Cabe recordar aquí un punto negro de la historia de la Unión Europea: bajo la presión

1 Fernando Simón Yarza, "Sobre el artículo 155 de la Constitución," El País, October 23, 2017, https://elpais.com/elpais/2017/10/22/opinion/1508674821_627304.html.

2 Camilo S. Baquero, "El nuevo Gobierno catalán toma posesión y pone fin a la intervención de la autonomia," El País, June 3, 2018, https://elpais.com/ccaa/2018/06/02/catalunya/1527933778_813805.html.

3 Fontelles et al. 2017, 22-26.

4 Busygina and Zakharov 2009, 191-192.

5 Volkova 2014, 296-297. 
de EE. UU., la UE tuvo que reconocer la independencia de Kosovo, a pesar que no hubo un referéndum oficial sino nominativo. Esto, sumado a otras situaciones de indole parecida, permite hablar de una especie de política de "doble rasero" a otros países. En general, puede decirse que los acontecimientos de la Unión tras la caída del Muro de Berlín en 1989 fueron hasta cierto punto contradictorios y polémicos: se modificó todo el mapa de Europa, desde la reunificación alemana hasta la desintegración de Yugoslavia y Checoslovaquia. La disolución de la Unión Soviética también jugó un papel importante desde el punto de vista geopolítico. Como resultado de este proceso surgieron 15 países sin experiencia en la construcción del estado y el sistema estatal, muy dependientes de la URSS, que se encontraron en una situación económica y social muy difícil. Con el paso de los años, o de décadas, la situación se fue normalizando en países como Bielorrusia, Kazajistán, Armenia y Rusia. Este tipo de problemas también se da en territorios ocupados, como la República Turca del Norte de Chipre. Al mismo tiempo, a menudo se repite la tesis de la firmeza de las fronteras como garantía de seguridad de la Unión Europea.

La Constitución de la Unión Europea establece que si la nueva región independiente de uno de sus Estados miembros quiere incorporarse a la Unión, es obligatorio el consenso de dicho Estado.' De este modo, la Unión Europea limita considerablemente la posibilidad de independencia de las autonomías y las regiones que quieran separarse, ya que al no formar parte de la UE pierden automáticamente los privilegios y beneficios de la Unión, como el libre tráfico de mercancías, personas y capitales, y esto afectaría directamente a su economía, que sería más vulnerable y débil, con la obligación añadida de adoptar una nueva moneda distinta al euro, etc. ${ }^{2}$ Además, los políticos españoles no apoyan la iniciativa de los catalanes, sino que se pronuncian a favor de la integridad del estado. Por otra parte, cabe resaltar el marcado espíritu de los españoles respecto al patriotismo y al concepto de una España unida, un país formado por 17 autonomías que conciben como el resultado de un proceso histórico al que no están dispuestos a renunciar, por lo tanto se muestran contrarios en general a la separación de las regiones más nacionalistas del país, que debe permanecer unido. Observamos, por tanto, una postura contraria al fenómeno del separatismo tanto a nivel político como a nivel social.

En el caso del referéndum el 1 de octubre de 2017, los países de la Unión Europea expresaron su apoyo al gobierno de España y subrayaron que el referéndum era ilegal. Además, mencionaron que el problema debía resolverse a través del diálogo entre las partes y con arreglo a la Constitución española. Una de las primeras personas que se pronunció a favor del gobierno oficial de España fue el presidente del Consejo Europeo (CE), Donald Tusk, que indicó que el gobierno central de Madrid sería "el único interlocutor" de la Unión Europea. ${ }^{3}$ De la misma manera, el presidente del Parlamento Europeo señaló que la legalidad es la base del diálogo con la Unión Europea y que ninguno de los países europeos reconocería la declaración de independencia de Cataluña. ${ }^{4}$

Con anterioridad a la celebración del referéndum, los representantes regionales de Cataluña trataron de recabar apoyos de la Unión Europea, sin éxito. La postura de la UE frente a los problemas de Cataluña fue un varapalo para las autoridades catalanas y, a la vez, fortaleció la postura del gobierno oficial de España. En estas circunstancias, los representantes catalanes decidieron defender su postura mediante una exposición

1 "Tratado por el que se establece una Constitución para Europa," Unión Europea, accessed October 1, 2020, https://europa.eu/european-union/sites/europaeu/files/docs/body/treaty_ establishing_a_constitution_for_europe_es.pdf.

2 Klochko 2012, 90-121.

3 "Sueño separatista en peligro: el mundo se opone a un Estado catalán," HispanTV, October 27, 2017, http://www.hispantv.com/noticias/espana/357827/reaccionesinternacionales-independencia-cataluna-parlament.

4 lbid. 
mediática muy activa e influyente en la opinión pública internacional.' La postura de la Unión Europea es clara y evidente: no desea establecer nuevos precedentes. El expresidente de la Comisión Europea, Jean-Claude Juncker, señaló que se trata de un asunto interno de España; por tanto, la solución depende sólo de España y, en cualquier caso, el nuevo estado no formaría parte de la Unión Europea. Además, alentó a las partes a iniciar un diálogo abierto y subrayó que la violencia nunca contribuye a resolver conflictos. ${ }^{2}$ En general, los países europeos y latinoamericanos también afirmaron con rotundidad que España debía tomar medidas para desescalar el conflicto con esta comunidad autónoma. Toda la responsabilidad de la solución de la crisis recayó en España ${ }^{3}$, que reaccionó con rigidez, utilizó métodos radicales y prohibió huelgas y manifestaciones, desestimando el diálogo abierto con los representantes de Cataluña.

Cabe agregar que, en la actualidad, se observa en el mundo una tendencia más marcada hacia la unidad que hacia el separatismo. Son corrientes que, sin ser necesariamente contrarias al separatismo, pueden explicarse como un deseo de los países de conservar el status quo, o de buscar una salida económica y política ventajosa frente a una situación dada. Esta tendencia a la unidad se observa también a nivel regional. Por ejemplo, en América Latina hay organizaciones como UNASUR, ALBA, CELAC, etc., que representan estos intereses de unidad; en África, la Unión Africana; en Oriente Medio, la Liga Árabe. Los principios de unidad se han fortalecido, aunque también hay tendencias separatistas. Un ejemplo muy actual es la situación de Reino Unido, con un referéndum en el que la población votó la salida de Reino Unido de la Unión Europea, lo que resultó sorprendente incluso para algunos politólogos y políticos británicos.

El problema de la Unión Europea reside en la crisis actual de la civilización europea. ${ }^{4}$ Por lo general, los procesos de desintegración de Europa son por causa de un alejamiento de los valores tradicionales, la liberalización de su cultura y la debilitación de los valores religiosos. Además, la Unión afronta serios problemas relativos a la desigualdad del desarrollo económico entre los países del sur y del norte, el envejecimiento de la población y la situación actual de la pandemia de coronavirus que padece el mundo entero, si bien cada país lo afronta a su manera, incluso dentro de la Unión Europea, lo que contribuye a disminuir la confianza de sus Estados miembros. Como es natural, todo ello influye en la situación de la Unión Europea y su política, y dificulta la relación entre los estados.

\section{Independencia de Cataluña: consecuencias posibles para la comunidad autónoma}

En la última parte del artículo analizamos teóricamente las posibles consecuencias de la independencia de Cataluña y los numerosos problemas que tendría que superar, llegado el caso. En lo que respecta a la consulta participativa de 2014 y el referéndum por la independencia de Cataluña de 2017, la comunidad autónoma menciona el precedente de Kosovo. Si Kosovo puede hacerlo, ¿por qué no podemos nosotros? Sin embargo, las autoridades catalanas desestimaron varios factores fundamentales, así como los riesgos y dificultades que atravesaría la comunidad. En primer lugar, la imposibilidad de ser parte integrante de la UE dado que España declaró oficialmente que no aceptaría a Cataluña y

1 Matveeva and Ol'shanskaya 2020, 153

2 "Statement on the events in Catalonia," European Commission, October 2, 2107, https://ec.europa.eu/commission/presscorner/detail/en/STATEMENT_17_3626.

3 Garcia 2019, 13-60.

4 Попов В. Кризис европейской цивилизации [Электронный ресурс] // Независимая газета. 14 ноября 2011 г.

URL: https://nvo.ng.ru/dipkurer/2011-11-14/10_crisis.html (дата обращения: 01.10.2020). 
tampoco está claro que permita la celebración de un referéndum legal. En segundo lugar, la necesidad de adoptar una moneda nueva, dado que quedaría excluida de la zona euro en caso de independizarse. En tercer lugar, Cataluña tendría que afrontar serias dificultades económicas debido, en parte, a la falta de demanda de productos catalanes fuera de España. En la actualidad, la mayor parte de la demanda de los productos de esta comunidad autónoma procede del país, más que de la Unión Europea. Por tanto, la creación del nuevo estado independiente exige la búsqueda inmediata de nuevos mercados para sus productos, lo que puede resultar difícil si consideramos que podría perder las prestaciones arancelarias de la Unión Europea. La producción orientada a la exportación podría ser deficitaria y por lo tanto la rentabilidad de la mercancía se vería reducida, ${ }^{1}$ lo que tendría efectos muy negativos en la economía de Cataluña. En cuarto lugar, es importante tener en cuenta que los problemas económicos influirán sin duda en otras esferas, sobre todo el turismo, que será menos atractivo debido al aumento de los precios. Al mismo tiempo, la economía de España también sufriría en gran medida ante la pérdida de las contribuciones de Cataluña en muchas regiones del país, como ya hemos mencionado anteriormente.

El fenómeno del separatismo resulta muy preocupante para la Unión Europea porque conduce a la ruptura de las relaciones económicas, la desaceleración general de la economía, la inestabilidad social y porque podría servir de precedente para procesos separatistas futuros. En la Unión Europea se observan varias tendencias separatistas, además de la situación de Cataluña: en Bélgica hay un nacionalismo no explícito, igual que en la región noreste de Italia, cuya agricultura sufrió un serio revés tras la aplicación de las sanciones a Rusia, etc. En este contexto, para la UE es de vital importancia establecer políticas dirigidas a consolidar la autoridad de los Estados miembros, pese a que las tendencias actuales puedan demostrar lo contrario. Además, la Unión Europea debería abandonar la llamada política de "doble rasero" y tener en cuenta la experiencia pasada en su futuro accionar.

\section{$\rightarrow$ Referencias / References}

Fontelles, Josep Borrell, Francesc de Carreras, Juan-José López Burniol, and Josep Piqué. Escucha, Cataluña. Escucha, España: Cuatro voces a favor del entendimiento y contra la secesión [Listen, Catalonia. Listen, Spain: Four voices for understanding and against secession]. Madrid: Ediciones Península, 2017. 336 p. [In Spanish]

Garcia, Romualdo Bermejo. "La Crisis Catalana y el Desgobierno de los Gobiernos de España" [The Catalan crisis and the mismanagement of the governments of Spain]. Anuario Español de Derecho Internacional 35 (2019): 13-60. https://doi.org/10.15581/010.35.13-60. [In Spanish]

Solé Tura, Jordi. Catalanismo y revolución burguesa [Catalanism and the Bourgeois Revolution]. Madrid: Cuadernos para el Diálogo, 1970. [In Spanish]

Walker, Neil. "Internal enlargement in the European Union: Beyond legalism and political expediency." In Secession from a Member State and Withdrawal from the European Union, edited by Carlos Closa, 32-47. Cambridge: Cambridge University Press, 2017. https://doi.org/10.1017/9781316771464.003.

Weiler, J.H.H. "Secessionism and its discontents." In Secession from a Member State and Withdrawal from the European Union, edited by Carlos Closa, 12-31. Cambridge: Cambridge University Press, 2017. https://doi. org/10.1017/9781316771464.002.

Бусыгина, И.М., Захаров, А.А. Общественно-политический лексикон. - М.: МГИМО-Университет, 2009. 276 c. [Busygina, I.M., Zakharov, A.A. Obshchestvenno-politicheskii leksikon [Social-Political Lexicon]. Moscow: MGIMO-University, 2009. 276 p.] [In Russian] 
Вербицкая, T.B. et al. Каталонский конфликт в Испании: последствия для Европейского Союза // Вестник Кемеровского государственного университета. Серия: Политические, социологические и экономические науки. - 2018. - №4. - С. 13-18. [Verbitskaya, T.V. et al. "Katalonskii konflikt v Ispanii: posledstviya dlya Evropeiskogo Soyuza" [The Catalan conflict in Spain: consequences for the European Union]. Vestnik KemGU. Series: Political, sociological and economic sciences 4(2018): 13-18.] [In Russian]

Волкова, Г. Испания и регионы. Политические вызовы и приоритеты XXI века. - М.: МГИМО-Университет, 2014. - 336 c. [Volkova, G. Ispaniya i regiony. Politicheskie vyzovy i prioritety XXI veka [Spain and the regions: the political challenges and priorities of the 21st century]. Moscow: MGIMO-University, 2014. 336 p.] [In Russian]

Клочко, Е. Испания и Европейский союз. Политическое измерение. - М.: Вебов, 2012. - 121 с. [Klochko, E. Ispaniya i Evropeiskii soyuz. Politicheskoe izmerenie [Spain and the European Union. The political dimension]. Moscow: Vebov, 2012. 121 p.] [In Russian]

Матвеева, Н.Ю., Ольшанская, Е.В. Социально-политические смыслы сторон испано-каталонского конфликта сквозь призму твиттер-коммуникаций // Сравнительная политика. - 2020. - №11(1). - С. 151-169. [Matveeva, N.YU., Ol'shanskaya, E.V. "Sotsial'no-politicheskie smysly storon ispano-katalonskogo konflikta skvoz' prizmu tvitter-kommunikatsii" [Socio-political meanings of the parties to the Spain-Catalan conflict through the prism of twitter communications]. Comparative Politics Russia 11, no. 1 (2020): 151-169.] https://doi.org/10.24411/2221-3279-2020-10012. [In Russian]

Небасуй, М.С. Угроза безопасности стран ЕС в аспекте каталонской борьбы за независимость // Архонт. - 2017. - №3. - С. 13-17. [Nebasui, M.S. “Ugroza bezopasnosti stran ES v aspekte katalonskoi bor'by za nezavisimost" " [Threat to the security of the EU countries in the aspect of the Catalan struggle for independence]. // Arhont. 3 (2017): 13-17.] [In Russian]

Хенкин, С.М. Сепаратизм против государства: случай Каталонии // Полис. Политические исследования. - 2018. - №3. - С. 166-175. [Khenkin, S.M. "Separatizm protiv gosudarstva: sluchai Katalonii" [Separatism versus the state: the case of Catalonia]. Polis (Russian Federation) 3(2018): 166-175.]

https://doi.org/10.17976/.jpps/2018.03.11. [In Russian] 


\section{The position of the European Union towards separatism in Catalonia}

\section{A.V. Makarycheva}

\section{$\rightarrow$ Abstract}

Secessionist sentiments are more common for the European Union than for other world regions. This tendency takes place to a large extent because of a crisis in the European values and religious traditions, and new priorities. Catalonia, as well as the Basque Country, tries to hold a direct dialogue with Brussels bypassing Madrid, which is accompanied with numerous difficulties: a discontent and warnings on the part of the official government of Spain, the necessity for the European Union to take into consideration the position of its member-state and many others. Despite the fact that regions started to play a more significant role in the European Union agenda, it still continues to follow the policy of a cautious attitude towards the autonomy separatism. Moreover, the EU tries to limit capabilities of the further existence and development of autonomies as independent states by institutional means. In addition, after the separation, a state is not yet a member of the European Union - it has to create new currency, it faces some economic problems. Given all these factors, autonomies will think twice before organizing a referendum, which is also difficult to hold, because it contradicts the Constitution of Spain.

\section{$\rightarrow$ Keywords}

Nationalism in Catalonia, the European Union, integration and disintegration processes in Europe, separatism in Spain, referendum in Catalonia

Disclosure statement: No potential conflict of interest was reported by the author.

https://doi.org/10.46272/24093416-2020-8-2-65-75

\section{Research article}

Anna Vladimirovna

Makarycheva, PhD in Political Science, Research Assistant at the Laboratory of International Trends Analysis, Lecturer at the Spanish Department, Moscow State Institute of International Relations (MGIMO-University) (Russia)

\section{E-mail:}

makarycheva.a.v@my.mgimo.ru

For correspondence: 119454 Russia, Moscow, av. Vernadskogo, 76

For citation: Makárycheva, Ánna Vladimirovna. "La postura de la Unión Europea frente al separatismo en Cataluña" [The position of the European Union towards Separatism in Catalonia]. Cuadernos Iberoamericanos 8 , no. 2 (2020): 65-75. https://doi. org/10.46272/2409-3416-20208-2-65-75. [In Spanish]

Received: 02.09.2020

Accepted: 05.10.2020 


\section{Позиция Европейского Союза по вопросу каталонского сепаратизма}

\section{А.В. Макарычева}

\section{$\rightarrow$ Аннотация}

Сепаратистские тенденции более характерны для Европейского Союза (ЕС), чем для всего остального мира. Данный факт объясняется прежде всего кризисом европейских ценностей, религиозной составляющей и новыми приоритетами. Намерение Каталонии, как и Страны Басков, вести прямой диалог с Брюсселем без участия Мадрида сопряжено с многочисленными трудностями: ключевое препятствие представляют недовольство и предупреждения со стороны испанского правительства в контексте необходимости учета Европейским Союзом официальных позиций государств-членов. Несмотря на то, что регионы стали занимать более видное место в повестке дня ЕС, руководство этого интеграционного объединения внимательно следит за положением автономий и не выступает в пользу сепаратизма. ЕС пытается ограничить активность и преобразование автономий в независимые государства, используя институциональные меры. Помимо этого, новое независимое государство не войдет автоматически в европейское пространство, поскольку перестанет быть частью ЕС и поэтому будет вынуждено решать ряд вопросов, таких как создание новой валюты и борьба с возможным экономическим кризисом, в дополнение к потенциальному недовольству снижением уровня жизни и социальными потрясениями. Все эти факторы влияют на принятие решений о референдуме, особенно когда речь заходит об оценке возможных последствий таких действий. Следует отметить, что при подобных обстоятельствах автономия будет с особой внимательностью подходить к вопросу о референдуме, в частности и потому, что данные шаги противоречат Конституции Испании.

\section{$\rightarrow$ Ключевые слова}

Каталонский национализм, Европейский Союз, интеграционные процессы, референдум в Каталонии, сепаратизм в Испании, европейская дезинтеграция

Конфликт интересов: Автор заявляет об отсутствии потенциального конфликта интересов. https://doi.org/10.46272/24093416-2020-8-2-65-75

\section{Исследовательская статья}

\section{Макарычева Анно}

Владимировна, К.полит.н., научный сотрудник Лаборатории анализа международных процессов, преподаватель Кафедры испанского языка МГИМО МИД России, Москва (Россия)

E-mail:

makarycheva.a.v@my.mgimo.ru

Для корреспонденции: 119454 , Россия, Москва, просп. Вернадского, 76

Для цитирования: Makárycheva Ánna Vladimirovna. "La postura de la Unión Europea frente al separatismo en Cataluña" [The position of the European Union towards Separatism in Catalonia]. Cuadernos Iberoamericanos 8, no. 2 (2020): 65-75. https://doi. org/10.46272/2409-3416-20208-2-65-75. [In Spanish]

Статья поступила в редакцию: 02.09.2020

Принята к публикации: 05.10.2020 